\title{
Smart Leaf Recognition System
}

\author{
G. Sekar, P.M. Benson Mansingh
}

\begin{abstract}
One of major issue nowadays is the agricultural productivity which is something our Nation's economy highly depends. Technology based advancements may lead to detection of diseases in plants which are quite natural. Care should be taken in this area before it causes serious effects on plants which mainly affect the product quality, quantity or productivity. Early stage detection of diseases in plants through some automatic technique is beneficial as it reduces a huge work of monitoring in large acres of crops. When they appear on plant leaves, earlier detection helps us to increase the yield and productivity. This paper presents an algorithm for image processing technique which is used for automatic detection and classification of plant leaf diseases with the help of raspberry pi and sensors. This survey is about different diseases and its classification, techniques which are used for plant leaf disease detection and also its respective fertilizer sprayed on the leaves.

Keywords- Image recognition, Open CV, Raspberry pi and
\end{abstract} Ultrasonic sensors.

\section{INTRODUCTION}

In any field, it is very essential to recognize the disease for the anticipation of losses. Health monitoring and finding of plant disease is very acute to practice viable agricultural science. Most of the leaf diseases seen on the plant leaves will have visually recognizable shapes. The huge amount of processing time, field work and plant disease knowledge are required to implement a Leaf disease detection system. The pictures with two dimensional orientations, which represent the appearance of some, subject normally a person or a physical object. Digital image processing is used to process the images of leaves affected by disease by digital computers. These are the recent development in terms of human's attraction with graphical inducements. The fundamentals of Digital image processing are image acquisition, image enhancement, image restoration, color image processing, segmentation and image compression. Using Raspberry Pi automatic sprayer fertilizer technique is used to cure the particular plant leaf diseases. To detect the plant disease the plant leaf disease in very initial stage use of automatic disease detection technique is beneficial. The process of leaf detection is monitoring some basic features of leaf and then comparing with the values obtained in the available date sets. Leaf disease has been affecting many aspects in the field of agriculture mainly they are production, quality and quality. Leaf disease detection can be helpful for the farmers. The diagnoses of leaf diseases in plants are inadequate due to human visual capability [2]. However, this diagnosis method is time consuming and monotonous.

Revised Manuscript Received on May 15, 2020.

* Correspondence Author

Dr.G.Sekar*, Electronics and Communication Engineering-, Sri Ramakrishna Institute of Technology, Coimbatore, India. Email: gsekarganesh@gmail.com

Mr.P.M. Benson Mansingh, Electronics and Communication Engineering, Sri Ramakrishna Institute of Technology, Coimbatore, India. E-mail: benson.ece@srit.org

(C) The Authors. Published by Blue Eyes Intelligence Engineering and Sciences Publication (BEIESP). This is an open access article under the CC BY-NC-ND license (http://creativecommons.org/licenses/by-nc-nd/4.0/)
An automatic system which detects, classifies and automatically recognizes the plant leaf diseases is much needed to improve the quality. An automatic system based on Computer vision technique can be used to classify, recognize and detect plant leaf disease which affects the crops in agriculture fields. The growth of computer vision based automated system saves the consultation time of farmer's with the agriculture experts. Automatic leaf disease detection[3] is the main topic for agriculture research because it can manage the monitoring of large agriculture crop fields automatically and detects the disease from the plant leaves. Further, these automatic systems give the conveniences of capturing, storing, compressing and processing the images which are collected from agriculture fields.

\section{LITERATURE SURVEY}

Several research articles were studied and the survey has made based on it, one such article developed by Adam et al, phaeosphaeria leaf spot (PLS) is viewed as one of the real infections that debilitate the soundness of maize generation in tropical and subtropical African areas. The article about The Early Stage of Phaeosphaeria Leaf Spot Infestations in Maize Crop Using In Situ Hyper spectral Data and Guided Regularized Random Forest Algorithm. The goal of the present examination was to research the utilization of hyper spectral information in distinguishing the beginning time of PLS in tropical maize. Field information were gathered from solid and the beginning time of PLS more than two years (2013 and 2014) utilizing a handheld spectroradiometer.

Chaitali Awachar, Minal Chavan, Jyoti Dorage and Namrata Gawande et al, have been developed and these papers around $75 \%$ of Indians are agriculturists. They kept the title as Expert System for Agriculture Using Sensors and Image Processing Techniques. Yet at the same time a few agriculturists are following customary strategy for cultivating. The conventional techniques prompts illadvised, wasteful cultivating hones because of which, ranchers are not getting expected creation and along these lines it influences on special. Yet, utilizing master framework they can decrease the odds of these issues. In this way, we have examined existing master framework for agriculture. They will outline a specialist framework for farming which comprises of:

1) Testing of soil to decide soil quality and propose compost contingent upon soil quality.

2) Control plant sickness and develop the plants in solid condition. 


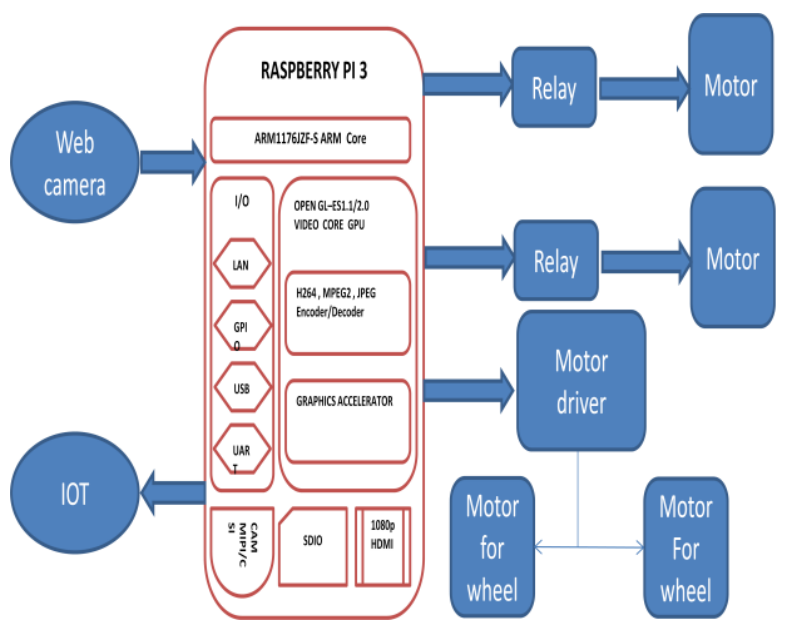

Fig. 1. Block diagram for proposed system

\section{OPEN CV}

Open CV is an open source computer vision library.This is active development on interface for python.Open $\mathrm{CV}$ was designed for computation efficiency and with a strong focus on real time application .In this Open CV the Algorithm used is SVM and Linear Binary Pattern. Digital image processing technique uses three types of images given as follows.

1.Binary image, 2. Gray scale image, 3.Color image

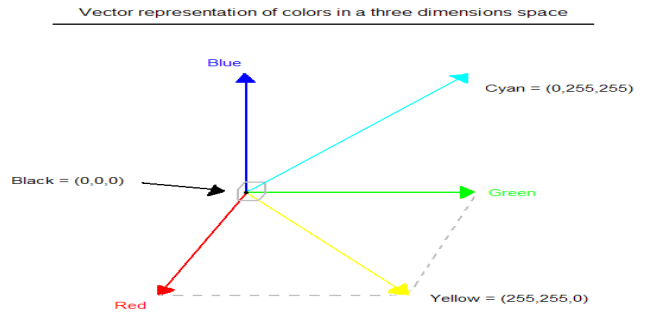

Fig. 2. Vector representation in Open CV

\section{ULTRASONIC SENSOR}

This uses sound instead of light for detection .The operation includes when an electric pulse with high voltage is applied to the ultrasonic transducer it vibrates in a specific spectrum of frequency and generate the burst of sound waves. If the object comes into the line of sight sound reflects as echo and an electric pulse is generated.

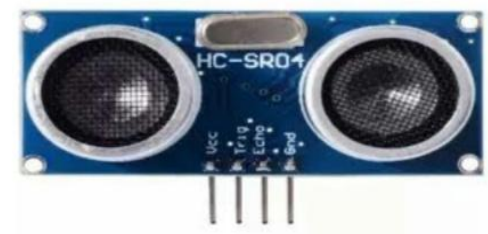

Fig. 3. Ultrasonic sensor

The distance is measured with the help of time taken between the transmission and reception.

\section{RASPBERRY PI}

The system-on-chip (SoC) multimedia processor Broadcom BCM2837 is used as a central processing unit of the Raspberry $\mathrm{Pi}$ system. The most important part of the
Raspberry Pi based system components such as Graphical Processing units, microprocessors, hardware equipments for audio and video communications are integrated as a single component built-in at the centre of the board under the memory space of the system $256 \mathrm{MB}$. However, the proposed SoC design based on the Broadcom BCM2837 is entirely different to the conventional processing units used in desktop or laptop computers. ARM processor, known as Reduced Instruction set computers is also used[1].

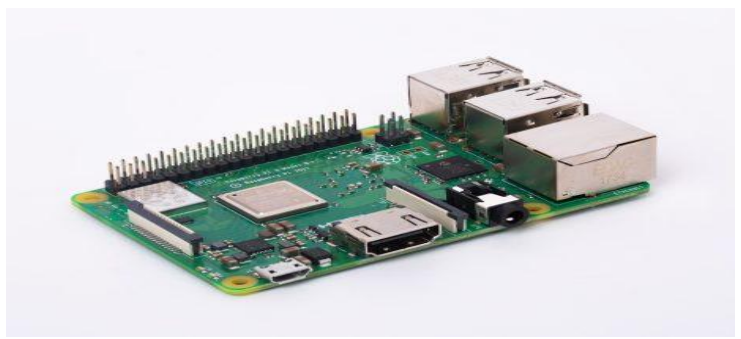

Fig. 4. Raspberry Pi

\section{RELAY AND MOTOR DRIVER}

The function a relay is, it can switch from one circuit to a second circuit which can be completely separate from the first. For instance, a relay can be used to switch a low voltage battery circuit to a $230 \mathrm{~V}$ AC mains circuit. The connection inside the relay is not electrical between the two circuits; the link inside the relay is magnetic and mechanical. Relays are very simple devices.

The motor driver IC L293D is a typical Motor driver which allows the DC motor to drive on both directions. L293D is a 16-pin IC which can control a pair of DC motors simultaneously in any directions, which is nothing but you can able to control two DC motors with a single L293D IC.

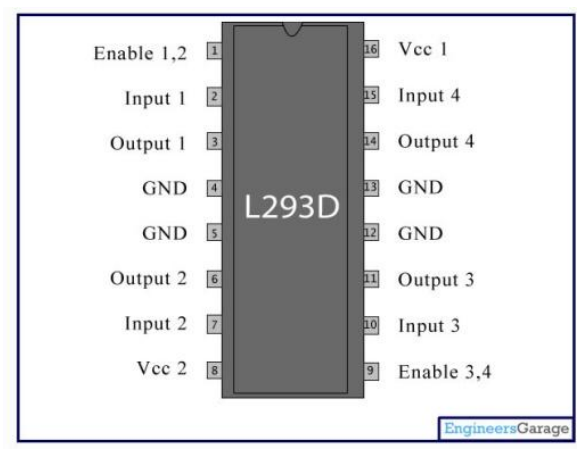

Fig. 5. Motor driver

\section{VII.PROPOSED ALGORITHM}

Step1: Initialize by capturing the image of the leaf Step2: Obtained image is checked by using the data given and decide whether it is affected by disease or not. Step3: While checking the leaves if it is a not an affected one it skip to the next leaves.

Step 4: If it is an affected one, it detect the disease and show the type of that disease and automatically uses the fertilizer by spraying on the affected leaf

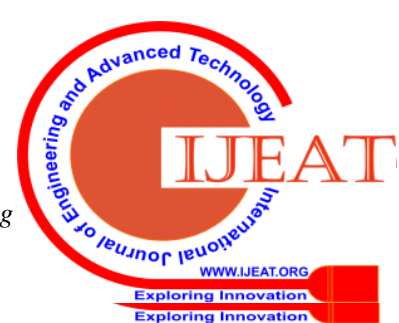




\section{EXPERIMENTAL SETUP}

The proposed work has two sections, one is software section and other is hardware section. In software section we use open CV for python. In python we have two main aspects, one is testing and other is training about the leaves. The main program tells about the automatic spraying method, distance calculation and direction of moving wheel movement [5]. In hardware section we use raspberry pi 3.Raspberry pi 3 is an advanced microprocessor. Web camera is used to capture the images. Motor is used for automatic spraying fertilizer. Motor driver is used to control the moving the wheel. Relays are used to control the motors. Ultrasonic sensor is used to measure the distance of the moving wheel. As a result, if it is an affected leaves it automatically sprayer the fertilizer in the defected leaves. If it a healthy leaf it moves to the next leaf [4].

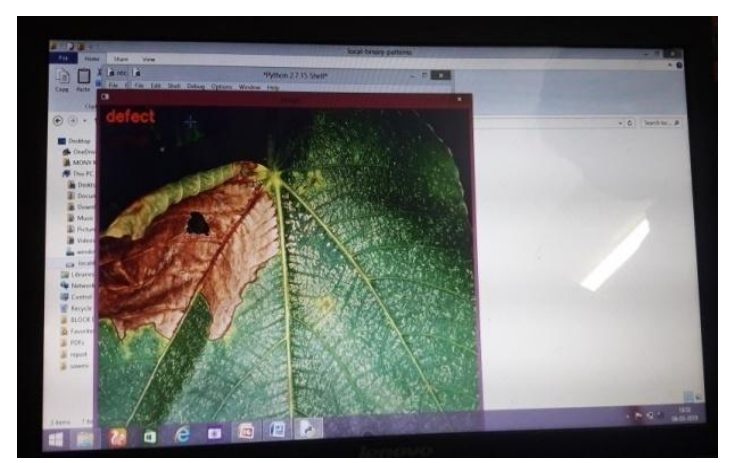

Fig. 6. Simulation result

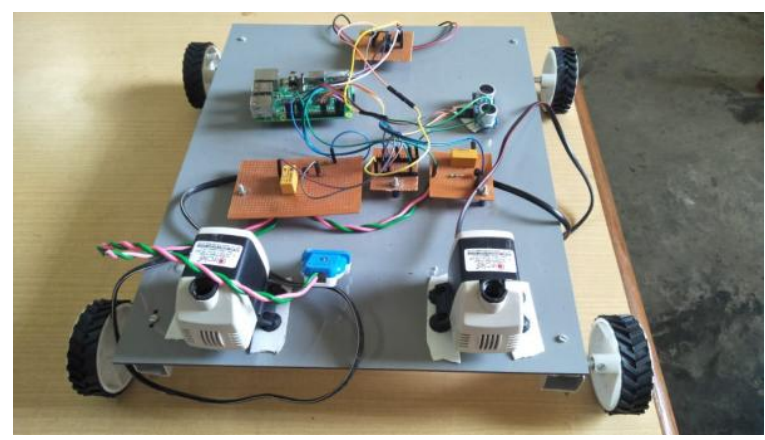

Fig. 7. Prototype model

\section{CONCLUSION}

The proposed system is very helpful for agriculturist because it is efficient as compared to the manual method. These systems widely used to replace the conventional leaf diseases recognition technique and also it is used by agricultural experts in identifying correct pesticide and its quantity to overcome the problem in an efficient and effective manner.

\section{ACKNOWLEDGEMENT}

The authors would like to express our sincere gratitude and hearty thanks to our SNR Sons Charitable Trust, Coimbatore and Dr.M.Paulraj, Principal, Sri Ramakrishna Institute of Technology, Coimbatore for providing all kind of technologies to complete this work.

\section{REFERENCES}

1. YeniHerdinyeni, MohammadIlhamJamaluddin, Tri section and VektorDewanto,' An Integrated Smart Surveillce System for Disease Monitor Tropical Plantation Forest”, IEEE $17^{\text {th }} 2017$ pp:1822-1826.

2. AakankshaRastogi, RitikaArora, Shanu Sharma, "Leaf Disease Detection and Grading using Computer Vision Technology \&Fuzzy Logic"

3. Haiguang Wang, Guanlin Li, Zhanhong Ma, XiaolongLi, "Image Recognition of Plant Diseases Based on Backpropagation Networks"

4. SaiKirthi Pilli1, BharathirajaNallathambi, Smith Jessy George, VivekDiwanji , "eAGROBOT- A Robot for Early Crop Disease Detection using Image Processing"

5. Geier,P.W."Management of insects pests,Annual Review of Entomology 11”,2015 pp832-836.

\section{AUTHORS PROFILE}

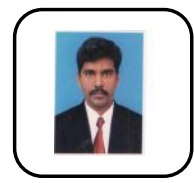

Dr. G. Sekar completed his Ph.D in Digital Image Processing under AnnaUniversity, Chennai. He obtained his M.E (Applied Electronics) Degree from Bannari Amman Institute of Technology, Sathyamangalam and his B.E degree in Electrical and Electronics Engineering from V.M.K.V Engineering College, Salem. $\mathrm{He}$ is having 12 years of teaching experience. He is currently working as an Assistant Professor (Senior Grade) in ECE department of Sri Ramakrishna Institute of Technology, Coimbatore. His research interest includes Digital Image Processing and Embedded systems. He has published eighteen papers in various reputed journals and conferences. He is a life member of IAENG.

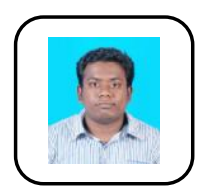

Mr. P. M. Benson Mansingh, started his graduation in the year 2006 in the faculty of Engineering, St.Peters engineering college, Chennai and obtained B.E (Electronics and Communication Engineering) in 2010. In 2011 he completed his CCNA Networking course (CISCO certified Network Associate) from NIIT, Chennai. He pursued his Post Graduate in the year 2012 at ANNA UNIVERSITY REGIONAL CAMPUS, Coimbatore under the faculty of Information and Communication Engineering and received the Master's Degree of Engineering in Network Engineering during the year 2014. Currently he is pursuing his Ph.D degree in ANNA UNIVERSITY, Chennai. He has a previous teaching experience of 4 years, $(2014-2018)$, in ANNA UNIVERSITY REGIONAL CAMPUS, Coimbatore (Government Institution). He has published three journals in Scopus and five journals in Thomson Reuters and has attended many international conferences. He is a member of IAENG. 